\title{
Phase Identification in Aged Catalysts Using STEM Depth Sectioning and Electron Energy-Loss Spectroscopy
}

Cheng-Han $\mathrm{Li}^{1}$ and Joerg Jinschek ${ }^{2}$

${ }^{1}$ The Ohio State University, Columbus, Ohio, United States, ${ }^{2}$ the Ohio State University, Columbus, Ohio, United States

Oxide-supported noble metal catalysts play significant roles in promoting the necessary catalytic reactions in our daily life, ranging from electrocatalytic reactions in fuel cells to exhaust gas emission reduction in the automotive industry. Among them, gamma-alumina supported rhodium ( $R$ h) nanoparticles automotive catalysts in the catalytic converter act as a key component to reducing the majority of nitric oxides emitted from the combustion engines. The scarcity of $\mathrm{Rh}$ and increasingly stringent emission regulations necessitate the deep understanding of mechanisms behind catalysts aging phenomenon.

The porous and semi-amorphous characteristics of industrial gamma-alumina support provide significant advantages such as high surface area and anchoring sites for catalysts nanoparticles. However, this makes it difficult to study the microstructure of the material under ideal imaging and spectroscopy conditions. Previous researchers have shown that alumina-supported $\mathrm{Rh}$-based catalysts $\left(\mathrm{Rh} / \mathrm{Al}_{2} \mathrm{O}_{3}\right)$ exhibit deactivation phenomena at elevated temperatures with various and highly debated explanations [1]-[6]. The ambiguity in such deactivation mechanisms is due to the limited information from the chosen method of characterization and the lack of direct atomic-scale visualization of the evolution of catalysts' microstructure. In this study, scanning transmission electron microscopy (STEM) and electron energyloss spectroscopy (EELS) are used to study the microstructure evolution of nanoparticles in industrial $\mathrm{Rh} / \mathrm{Al}_{2} \mathrm{O}_{3}$ catalysts upon accelerating aging treatments.

One challenge for imaging heterogeneous catalysts is the non-uniform substrate with highly dispersed metallic nanocatalysts that hinders the interpretation of the original structure based on 2D projections. In addition, aberration-corrected STEM enables sub-Angstrom spatial resolution but reduces significantly the depth of field due to the larger convergence angle. The effects of reduced depth of field will be more challenging for supported nanocatalysts. To visualize the three-dimensional information, STEM optical depth sectioning methods have been applied to reveal the microstructure of ideal semiconductors with dopants [7] and three-dimensional nanoparticles [8]. Newly formed phases with substitutional $\mathrm{Rh}$ atoms in gamma-alumina support under high-temperature oxidizing treatments will be revealed with depth sectioning STEM analysis to deeply understand the deactivation and regeneration phenomenon after environmental treatments. With the collections of focus series using aberrationcorrected ThermoFisher Themis Z STEM microscope, three-dimensional representation of microstructure will facilitate the interpretation of Rh-alumina interacting species with high-spatial resolution. In the meantime, in order to avoid microstructure alteration of beam-sensitive gamma-alumina supports, the lowdose analysis will be carried out and show the optimal conditions due to the trade-off between high contrast and minimum beam effects [9].

References

[1] H. C. Yao, S. Japar, and M. Shelef, "Surface interactions in the system RhA12O3," J. Catal., vol. 50, no. 3, pp. 407-418, 1977. 
[2] H. C. Yao, H. K. Stepien, and H. S. Gandhi, "Metal-support interaction in automotive exhaust catalysts: Rh-washcoat interaction,” J. Catal., vol. 61, no. 2, pp. 547-550, 1980.

[3] C. Wong and R. W. McCabe, "Effects of high-temperature oxidation and reduction on the structure and activity of Rh/A12O3 and Rh/SiO2 catalysts," J. Catal., vol. 119, no. 1, pp. 47-64, 1989.

[4] J. G. Chen, M. L. Colaianni, P. Chen, J. T. Yates, and G. B. Fisher, "Thermal behavior of a rhodium/alumina model catalyst: disappearance of surface rhodium upon heating," J. Phys. Chem., vol. 94, no. 12, pp. 5059-5062, Jun. 1990.

[5] R. W. Mccabe, R. K. Usmen, K. Ober, and H. S. Gandhi, "The Effect of Alumina Phase-Structure on the Dispersion of Rhodium/Alumina Catalysts," J. Catal., vol. 151, no. 2, pp. 385-393, 1995.

[6] D. D. Beck, T. W. Capehart, C. Wong, and D. N. Belton, "XAFS Characterization of Rh/A12O3 After Treatment in High-Temperature Oxidizing Environments," J. Catal., vol. 144, no. 1, pp. 311-324, 1993. [7] R. Ishikawa, S. J. Pennycook, A. R. Lupini, S. D. Findlay, N. Shibata, and Y. Ikuhara, "Single atom visibility in STEM optical depth sectioning," Appl. Phys. Lett., vol. 109, no. 16, p. 163102, Oct. 2016.

[8] R. Hovden, H. L. Xin, and D. A. Muller, "Extended Depth of Field for High-Resolution Scanning Transmission Electron Microscopy," Microsc. Microanal., vol. 17, no. 1, pp. 75-80, 2011.

[9] C.-H. Li, J. Wu, and J. Jinschek, "Revealing the Atomic Structure of Rh/ $\gamma$-Al2O3 Catalysts Using Low Dose Rate Electron Microscopy,” Microsc. Microanal., vol. 26, no. S2, pp. 3072-3074, 2020. 